\title{
Exodontia de segundo pré-molar impactado em mandíbula: relato de caso
}

\author{
Extraction of impacted second premolar in the mandibule: case report \\ Extracción de un segundo premolar impactado en la mandíbula: reporte de caso
}

\section{Resumo}

Dentes impactados são aqueles que não erupcionaram na cavidade oral e estão situados totalmente ou parcialmente no interior do osso, tal anomalia é multifatorial e ocorrem por fatores locais e sistêmicos. Os segundos pré-molares inferiores são a classe de dentes mais acometidas por impacções, excluindo os caninos superiores e os terceiros molares, na maioria das vezes encontra-se assintomático e o seu diagnostico se dá por exames de imagem. O objetivo do presente trabalho é relatar o procedimento de exodontia de um segundo pré-molar incluso em mandíbula, no qual estava impactado entre os dentes 34 e 36, para exodontia do elemento dental foi realizada uma incisão relaxante vestibular, osteotomia e odontosecção com broca em alta rotação, o elemento foi removido em fragmentos com auxílio de alavancas. O procedimento cirúrgico para exodontia do elemento impactado, neste caso, foi de significativa relevância, uma vez ponderado seus riscos e benefícios em relação as demais opções de tratamento. Sendo assim, a estratégia de abordagem utilizada apresentou sucesso na condução do caso clínico.

Palavras-chave: Dente pré-molar; Dente impactado; Mandíbula; Cirurgia bucal.

\begin{abstract}
Impacted teeth are those that didn't erupt in the oral cavity and are located totally or partially inside the bone. This anomaly is multifactorial and occurs due to local and systemic factors. Mandibular second premolars are the class of teeth most affected by impactions, excluding the upper canines and third molars, which in most cases are asymptomatic and are diagnosed by imaging exams. The objective of this article is to report the procedure of extraction of a second premolar included in the mandible, which was impacted between teeth 34 and 36. For extraction of the tooth was made a relaxing buccal incision, osteotomy and odontosection with drill in high rotation, removing it in fragments with the aid of levers. The surgical procedure for extraction of the impacted element, in this case, was of significant relevance, considering its risks and benefits in relation to other treatment options. Therefore, the approach strategy used was successful in conducting the clinical case.
\end{abstract}

Keywords: Premolar tooth; Impacted tooth; Mandible; Oral Surgery.

\section{Resumen}

Los dientes impactados son aquellos que no han erupcionado en la cavidad bucal y se encuentran total o parcialmente dentro del hueso, esta anomalía es multifactorial y se produce por factores locales y sistémicos. Los segundos premolares mandibulares son la clase de dientes más afectados por impactaciones, excluyendo los caninos superiores y terceros molares, la mayoría de las veces es asintomático y su diagnóstico se realiza mediante exámenes de imagen. El objetivo del presente trabajo es reportar el procedimiento de extracción de un segundo premolar incluido en la mandíbula, el cual fue impactado entre los dientes 34 y 36. Para la extracción del elemento dental una incisión bucal relajante, osteotomía 
y odontosección con fresa en alta rotación, el elemento se retira en fragmentos con la ayuda de palancas. El procedimiento quirúrgico para la extracción del elemento impactado, en este caso, fue de gran relevancia, considerando sus riesgos y beneficios en relación con otras opciones de tratamiento. Así, la estrategia de abordaje utilizada fue exitosa en la conducción del caso clínico.

Palabras clave: Diente premolar; Diente impactado; Mandíbula; Cirurgia bucal.

\section{Introdução}

Dentes impactados são aqueles que não erupcionam na cavidade oral em seu tempo previsto e permanecem situados totalmente ou parcialmente no interior do osso, com a conservação ou não do folículo pericoronário. A impacção de dentes permanentes é um fenômeno frequentemente encontrado com uma prevalência variável, estudos indicam que um em cada seis indivíduos com mais de 20 anos possuem um ou mais dentes impactados (Sandler \& Splingater, 1991; Alling \& Catone, 1993; Primo, Andrade, Oliveira \& Oliveira, 2011; Allaredy, Caplin, Markiewicz \& Meara, 2020).

A etiologia de tal anomalia é multifatorial, estando comumente associado a fatores locais e sistêmicos. Os fatores sistêmicos comumente observados são: deficiências endócrinas como o hipotireoidismo, displasia cleidocranial, e disostoses craniofaciais. Os fatores locais podem incluir discrepâncias severas de tamanho dental e de arco, falhas na reabsorção radicular na dentição primária, perda precoce dos dentes decíduos com redução de espaço no arco dental, presença de dentes supranumerários, trauma dento alveolar, anquiloses (Burch, Ngan \& Hackman, 1994; Frank, 2000; Primo et al., 2011; Allaredy et al., 2020).

Os segundos pré-molares inferiores são a classe de dentes mais acometidos pelas impactações, excluindo os terceiros molares e os caninos superiores. Estes dentes irrompem após os caninos, porém quando essa cronologia habitual não ocorre, geralmente, o segundo pré-molar encontra-se impactado (Collet, 2000; Abu-Hussein, Watted, Emodi \& Awadi., 2015; AlAbdallah., Al Hadidi, Hammad \& Dar-Odeh, 2018; Allaredy et al., 2020).

Os dentes impactados em sua maioria encontram-se assintomáticos e são notados geralmente por clínicos gerais ou ortodontistas, que fazem o diagnóstico (Filho, França, Araújo, Pereira, Belchior \& Sampieri, 2018; Mohammed, Mahomed \& Ngwenya, 2019). Comumente pode ser observada a desoclusão e a ausência dentária através de radiografias de rotina, como as periapicais (por meio de técnicas ortorradiais e suas variações para localização dentária), oclusais e panorâmicas (Clarck, 1910; Valente, Soares, Santos \& Silva, 2016; Filho et al., 2018). Atualmente, a tomografia computadorizada tem sido amplamente empregada para a localização e diagnóstico dos elementos dentários impactados. O elemento impactado pode se encontrar na face vestibular, lingual, alveolar, em posição horizontal, vertical ou angulada (Infante-Cossio,Hernandez-Guisado \& GutierrezPerez, 2000; Duarte, Figueiredo, Ramos, Esteves, Salazar, Martins \& Figueira, 2005; Kaczor-Urbanowicz, Zadurska \& Czochrowska et al., 2016; Ferraz, Carneiro, Stecke, Rayes \& Oliveira, 2019).

Os tratamentos a serem propostos variam de acordo com a possibilidade de permanência do elemento dental no arco, planejamento ortodôntico, presença de patologias associadas e proximidade com estruturas nobres. Sendo assim, são propostas, na maioria dos casos, a exodontia do elemento impactado ou o seu tracionamento ortodôntico (Kaczor-Urbanowicz et al., 2016; Allareddy et al., 2020).

\section{Metodologia}

Foi realizado um estudo intervencional, descritivo e qualitativo no formato de Relato de Caso do procedimento cirúrgico realizado. Os dados foram coletados por meio físico dos prontuários, após autorização da paciente e assinatura do Termo de Consentimento Livre e Esclarecido, conforme (Brown et al., 2016). O relato de caso, realizado com base em revisão de literatura, é apresentado com base nos descritores (Dente pré-molar, Dente impactado, Mandíbula; Cirurgia bucal). Para a execução do tratamento, respeitando os princípios éticos da Declaração de Helsinque, foi necessário a obtenção da assinatura do Termo de 
Research, Society and Development, v. 10, n. 14, e257101421939, 2021

(CC BY 4.0) | ISSN 2525-3409 | DOI: http://dx.doi.org/10.33448/rsd-v10i14.21939

Consentimento Livre e Esclarecido, pelo paciente, dispensando nesse caso a aprovação pelo Comitê de ética.

\section{Relato de caso}

Paciente do sexo feminino, melanoderma, 37 anos, hipertensa, procurou atendimento odontológico na Clínica Odontológica da Faculdades Integradas Aparício Carvalho-FIMCA de Porto Velho/RO para tratamento do dente 36, comprometido por extensa cárie e doença periodontal. Ao exame intraoral foi observada a ausência do elemento dental 35. A radiografia periapical evidenciou a presença do elemento 35 impactado, abaixo do dente 36. Sendo assim, foi solicitada uma tomografia computadorizada (Figura 1) que mostrou a localização do elemento dental 35 pela superfície lingual entre os dentes 34 e 36. O elemento dental 35 apresentava-se totalmente incluso e envolto por tecido ósseo e adjacente ao elemento 36, comprometido com carie extensa até a região de furca, além de comprometimento periodontal. Sendo assim, foi indicada remoção de ambos através de exodontia.

Figura 1- Radiografia panorâmica e cortes da tomografia computadorizada evidenciando a inclusão do elemento dental 35 entre os dentes 34 e 36 .

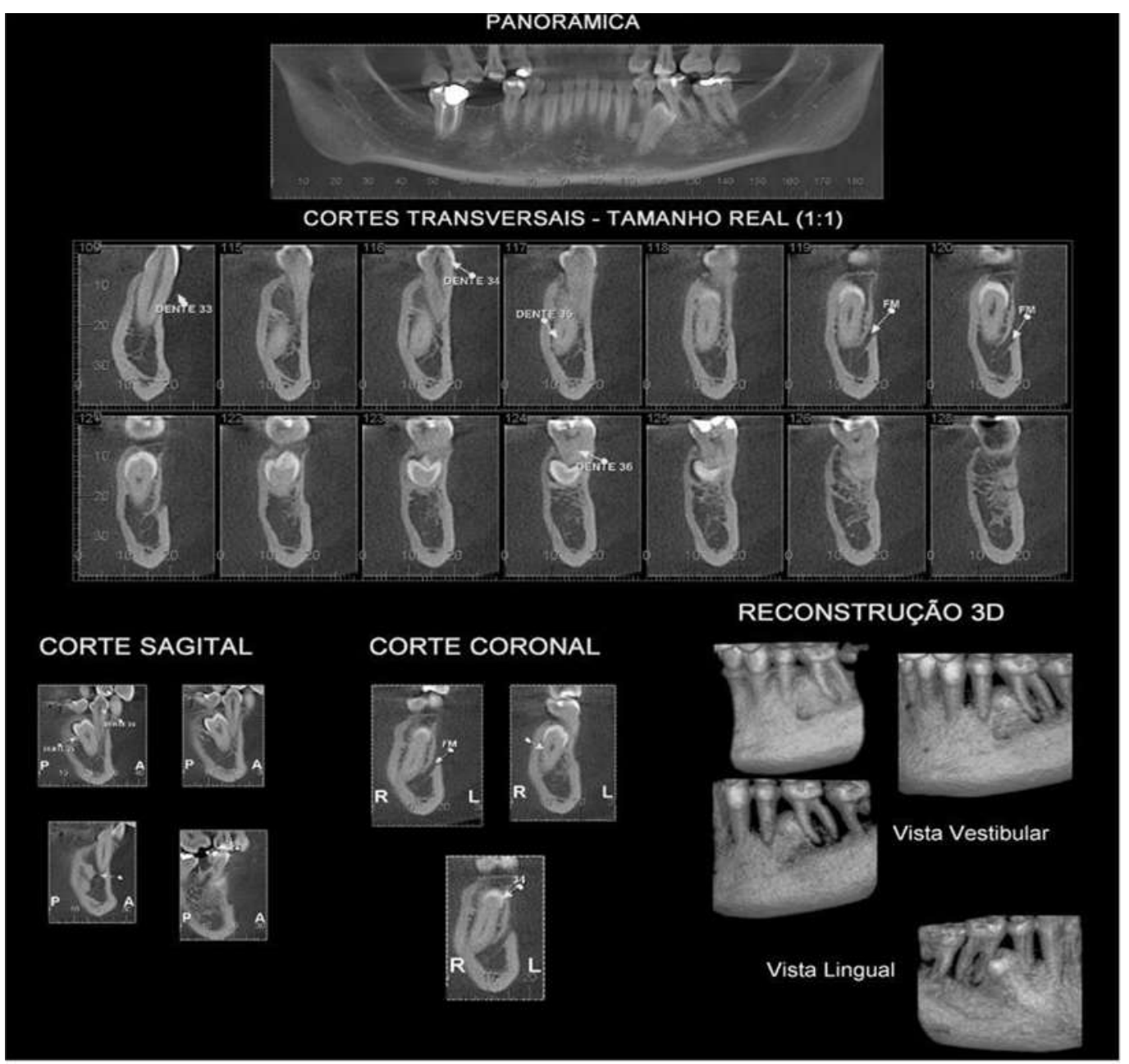

Fonte: Autores.

Com os exames clínicos e radiográficos, foi realizado o planejamento cirúrgico de exodontia dos elementos 36 erupcionado e comprometido, e 35 impactado, visando evitar complicações caso o elemento fosse mantido incluso. A medicação 
pré-operatória utilizada foi a dexametasona $4 \mathrm{mg}$ - um comprimido, amoxicilina 500mg- um comprimido, ambos uma hora antes do procedimento.

A antissepsia intra-oral foi feita com digluconato de clorexidina a 0,12\% e extra-oral com Clorexidina 0,2\% aquosa. Após o preparo e aposição dos campos cirúrgicos esterilizados, foi realizado o bloqueio dos nervos alveolar inferior, lingual, bucal e do nervo mentoniano esquerdo, com solução anestésica Mepivacaína 2\% com epinefrina 1:100.000. A seguir, procedeuse a sindesmotomia da gengiva na região dos dentes 36 e 34 com o descolador de Molt e posteriormente, a exodontia do 36 com auxílio de extratores e fórceps (Figuras 2 e 3).

Figura 2- Descolamento gengival do dente 36.

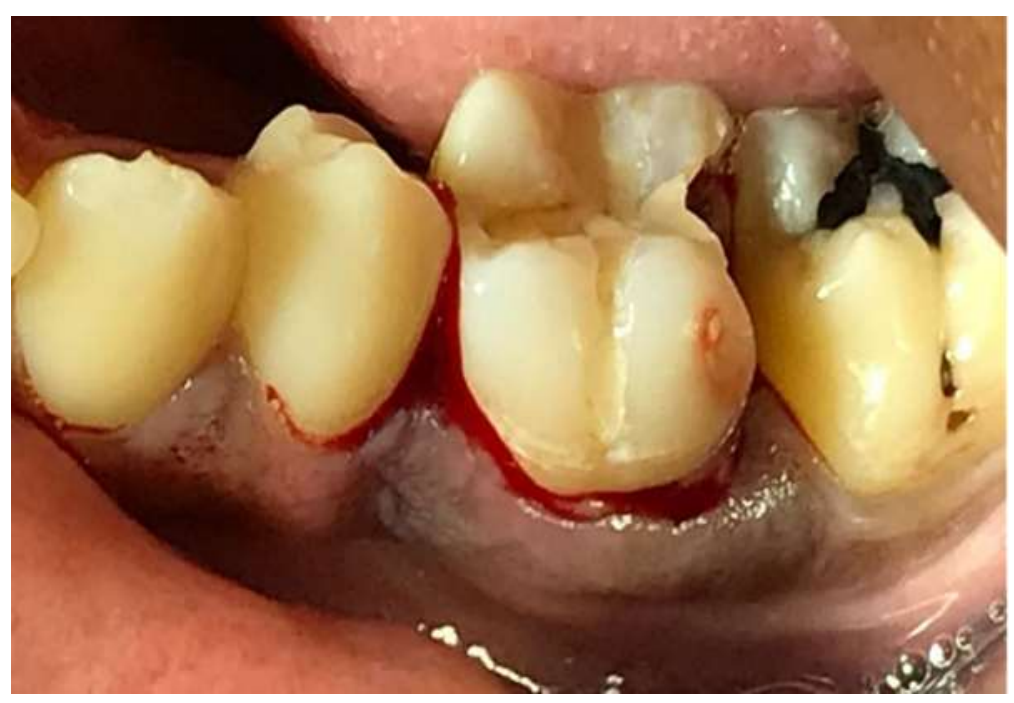

Fonte: Autores.

Para a exodontia do dente 35 foi adicionada uma incisão relaxante vestibular ao nível do elemento 34 com lâmina de bisturi 15C montada em cabo número 3 e (Figura 4).

Figura 3- Exodontia do elemento dental 36.

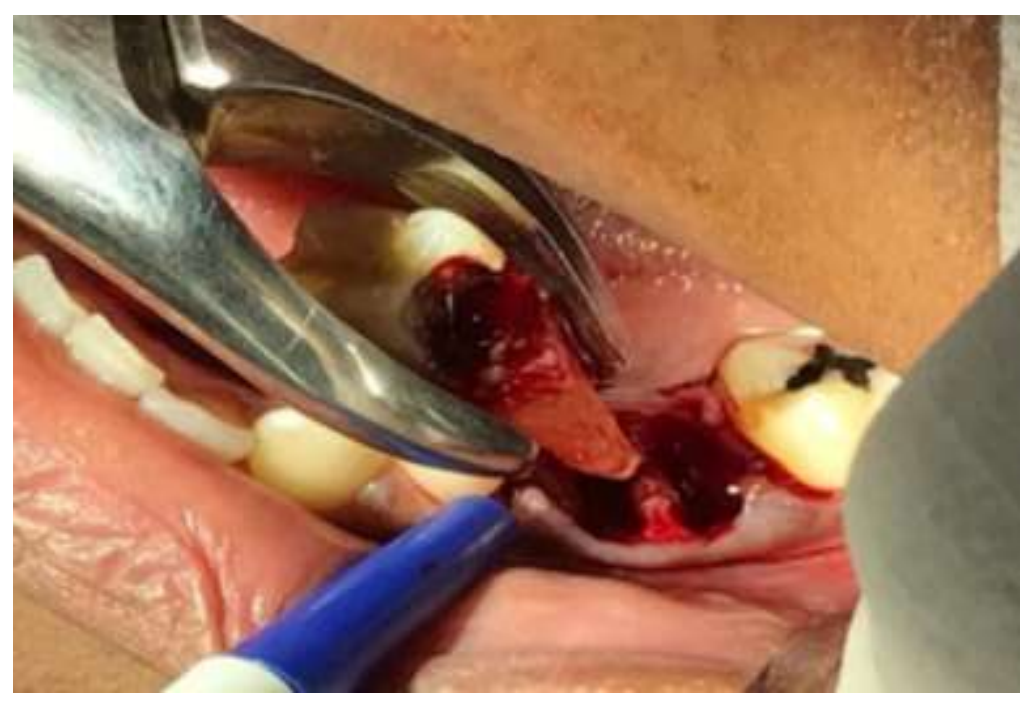

Fonte: Autores. 
Research, Society and Development, v. 10, n. 14, e257101421939, 2021

(CC BY 4.0) | ISSN 2525-3409 | DOI: http://dx.doi.org/10.33448/rsd-v10i14.21939

Figura 4- Realização da incisão e do retalho tipo envelope.

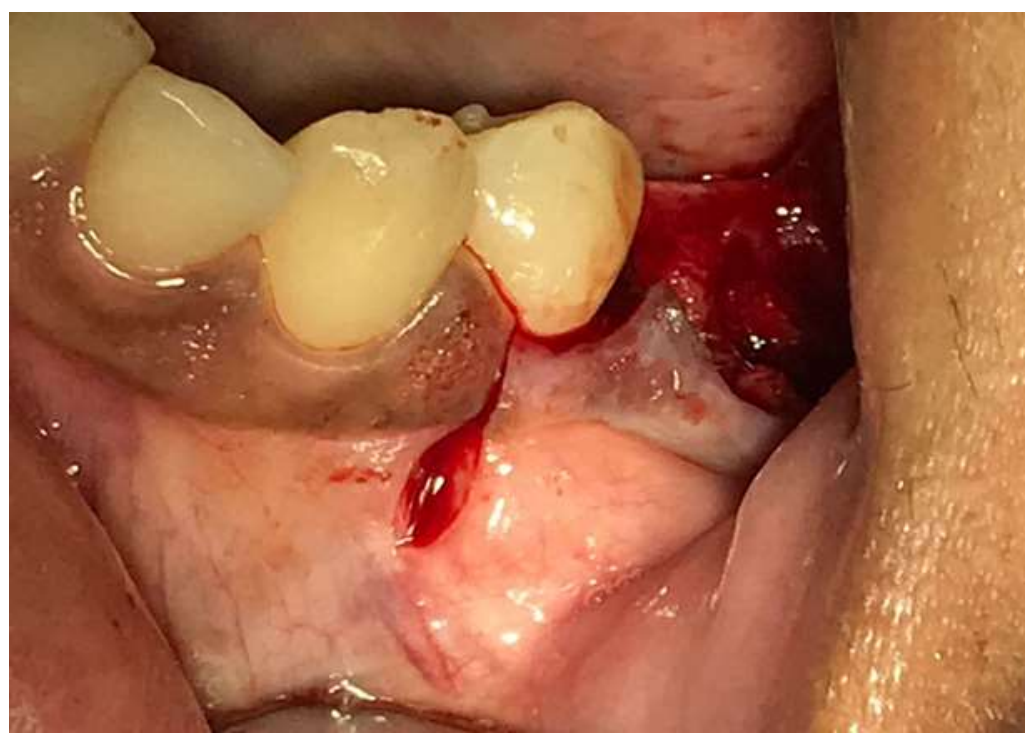

Fonte: Autores.

Para o descolamento muco-periosteal (Figura 5) foi utilizado o Molt 9, seguido de ostectomia vestibular com broca 702 de haste longa na caneta de alta rotação e com irrigação de cloreto de sódio a 0,9\% (Figuras 6).

Figura 5- Acesso cirúrgico após o descolamento muco-periosteal.

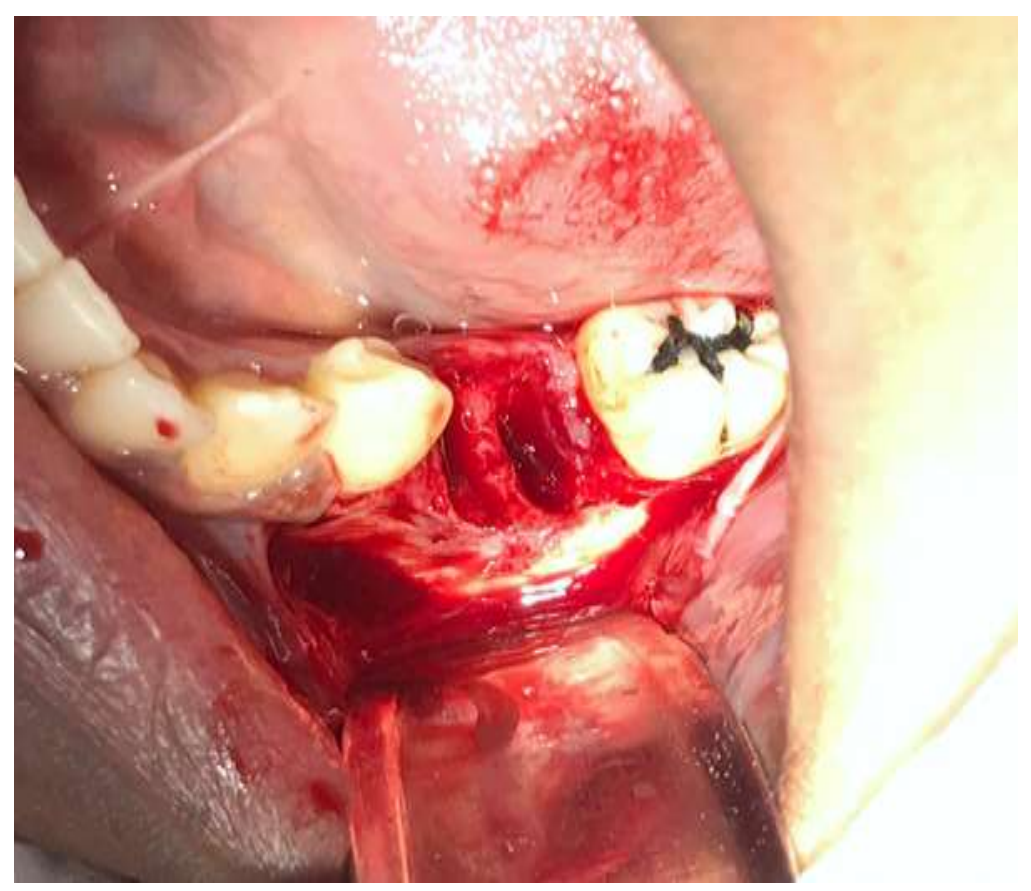

Fonte: Autores. 
Research, Society and Development, v. 10, n. 14, e257101421939, 2021

(CC BY 4.0) | ISSN 2525-3409 | DOI: http://dx.doi.org/10.33448/rsd-v10i14.21939

Figura 6- Osteotomia para exposição da coroa do elemento dentário.

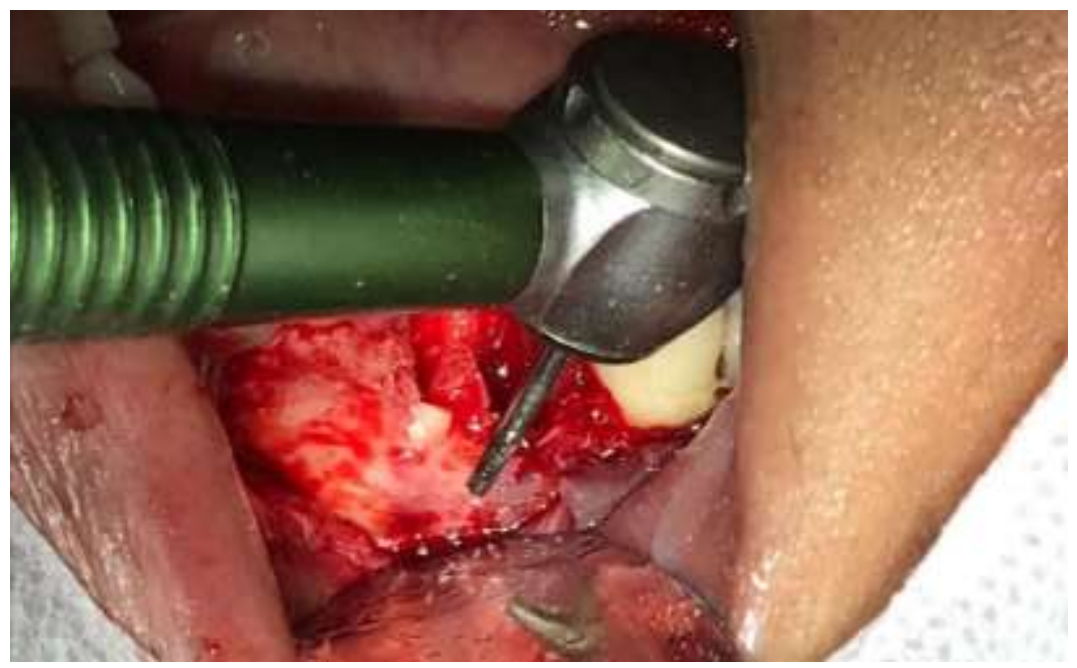

Fonte: Autores.

Logo após, foram feitas a odontossecção com broca 702, luxação e extração do dente com alavancas reta e apical, seguidas da curetagem do alvéolo (Figuras 7 e 8), acompanhada de irrigação abundante com soro fisiológico a $0.9 \%$.

Figura 7- Aspecto do alvéolo após exodontia do elemento dentário e curetagem do mesmo.

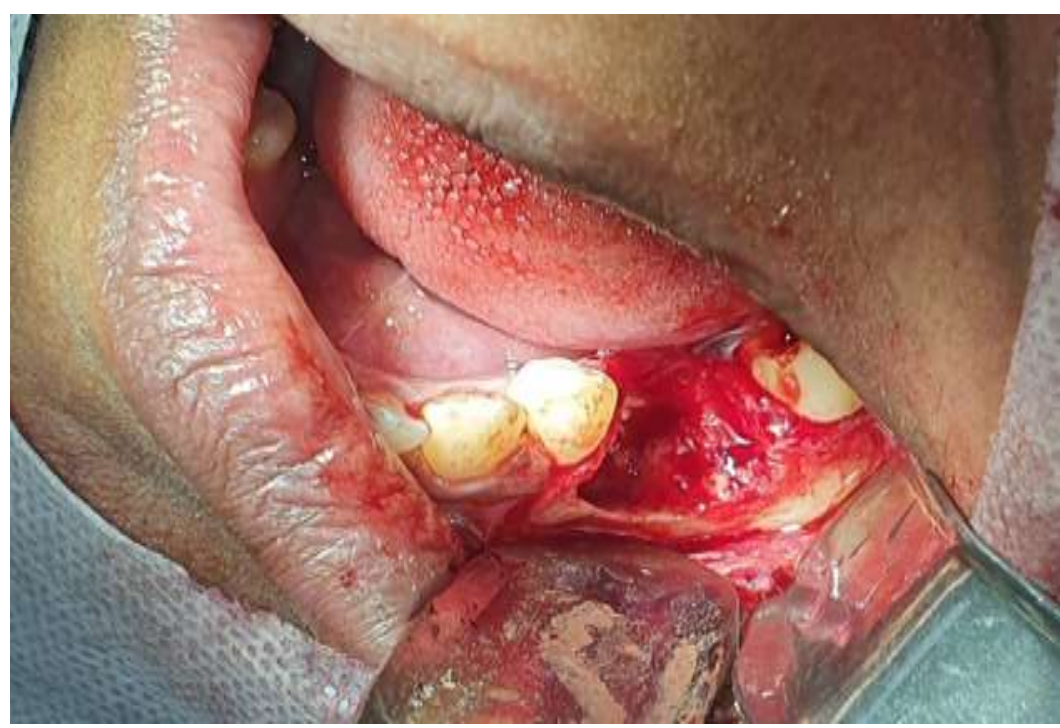

Fonte: Autores. 
Figura 8- Elementos dentais 35 e 36 após exodontia.

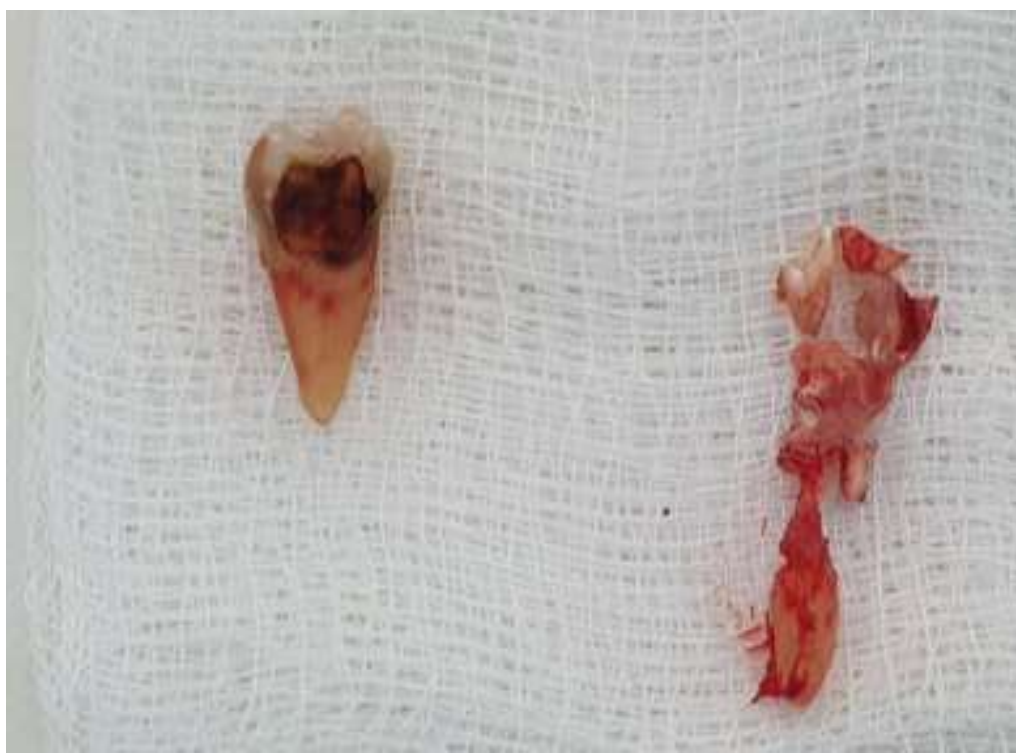

Fonte: Autores.

O procedimento cirúrgico seguiu sem intercorrências e foi finalizado com reposicionamento do retalho e sutura (Figura 9) com fio de Nylon 4-0 e agulha circular $3 / 8$ de $2,0 \mathrm{~cm}$.

Figura 9- Reposicionamento e sutura do retalho com fio de nylon 4-0.

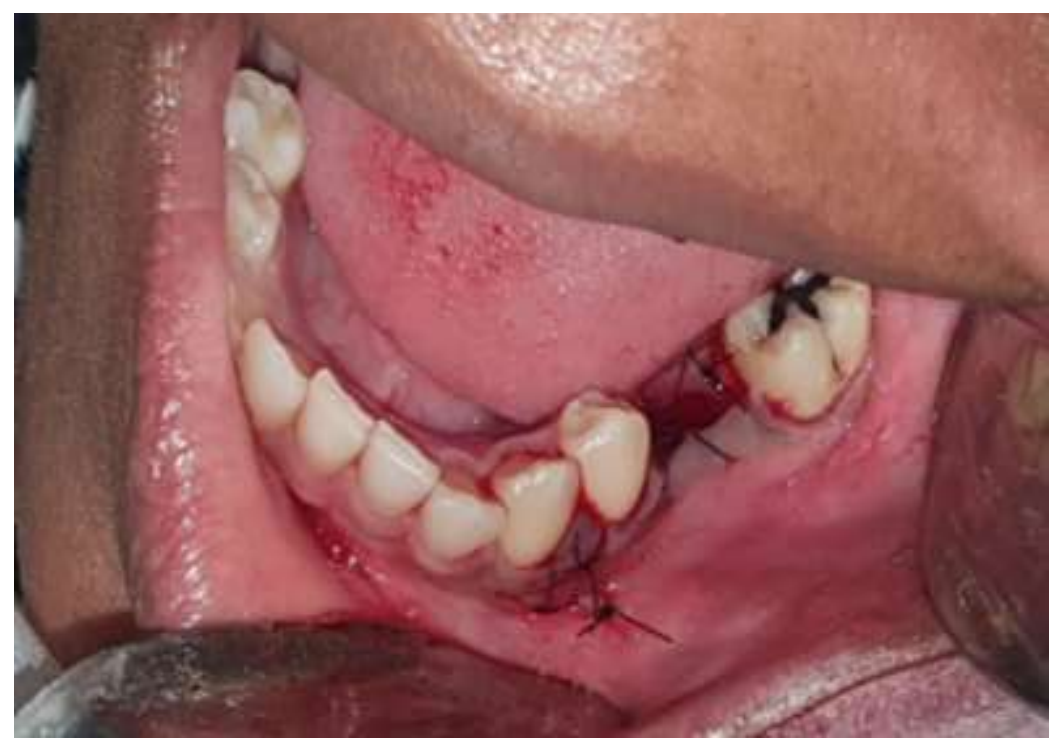

Fonte: Autores.

Após o procedimento, o paciente recebeu a prescrição medicamentosa de Amoxicilina de 500mg- 1 cápsula de 8/8h por 7 dias, Ibuprofeno 600mg- 1 comprimido de 8/8h por 2 dias, Dipirona sódica 500mg- 1 comprimido de 6/6h por 3 dias.

No décimo dia de pós-operatório a paciente retornou para reavaliação clínica, com remoção de suturas e radiográfica (Figuras 10 e 11). Em seu retorno ela relatou estar com parestesia na região mentual do lado esquerdo. Sendo assim, foi realizada a prescrição de ETNA- 1 comprimido, de 12 em 12 horas por 30 dias. Dentro desse tempo, a paciente referiu melhora gradativa da sensibilidade local. Decorrido tal período, a paciente relatou em nova reavaliação que a parestesia havia cessado. 
Research, Society and Development, v. 10, n. 14, e257101421939, 2021

(CC BY 4.0) | ISSN 2525-3409 | DOI: http://dx.doi.org/10.33448/rsd-v10i14.21939

Figura 10- Aspecto após remoção da sutura aos 7 dias de pós-operatório.

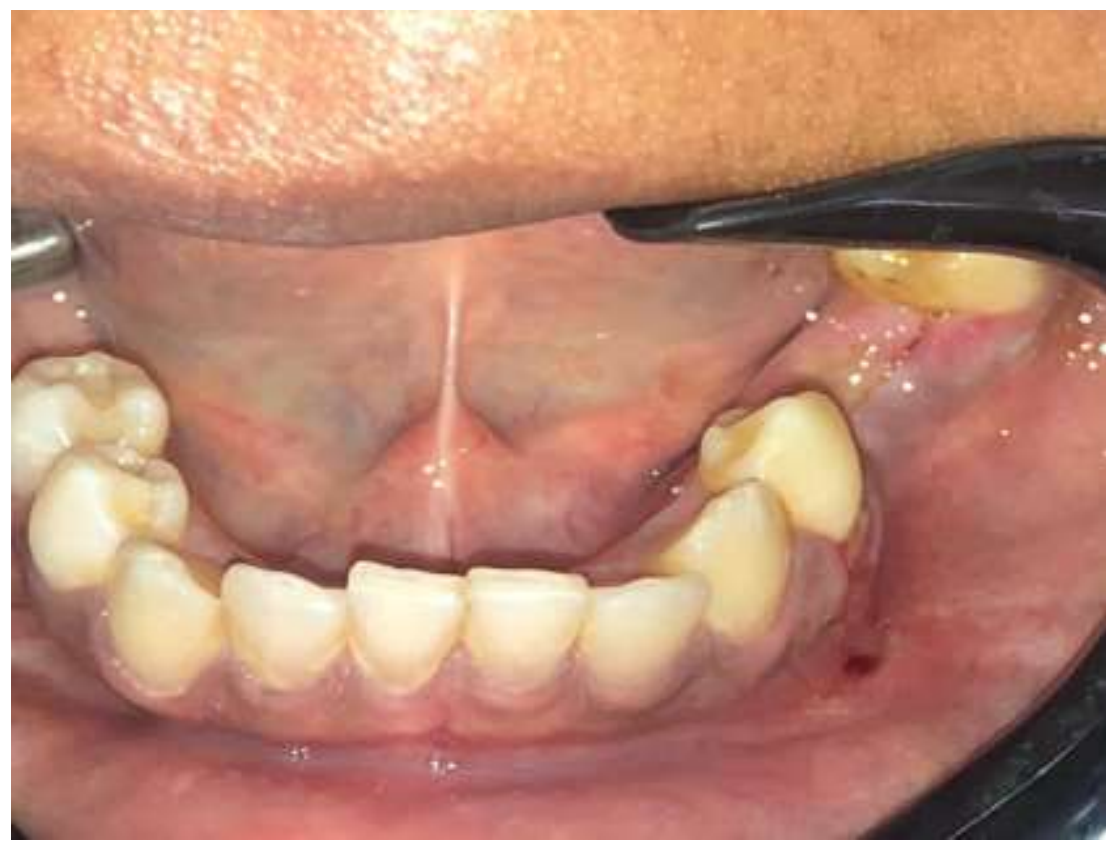

Fonte: Autores.

Figura 11- Radiografia panorâmica após 1 mês da exodontia evidenciando o reparo ainda em processo.

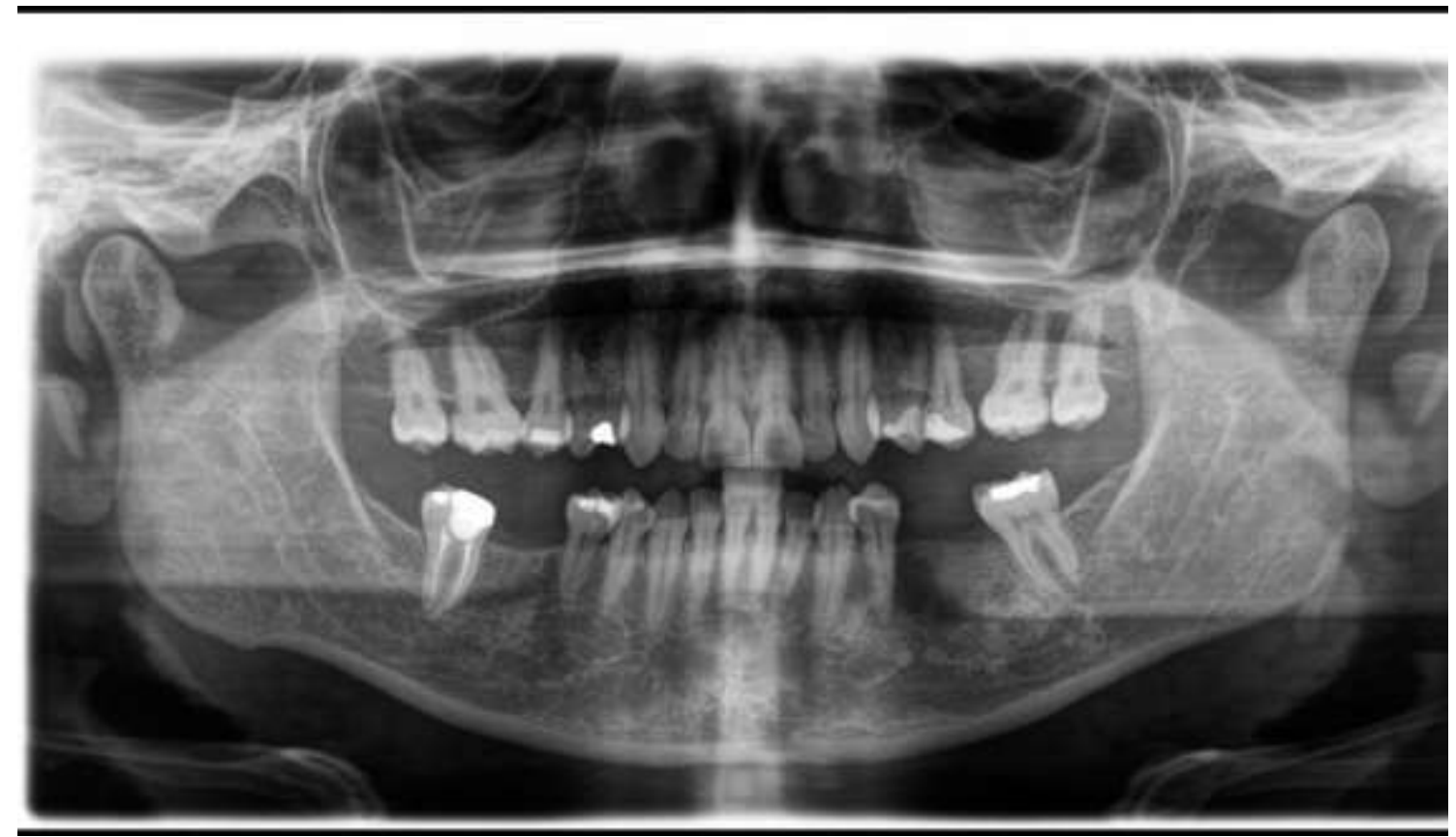

Fonte: Autores.

\section{Discussão}

As principais indicações para extração dentária incluem cárie dentária, doença periodontal, fraturas, falha no tratamento endodôntico, tratamento ortodôntico e protético, complicações de terceiros molares e solicitações do paciente, com custo e alternativas de tratamento associadas de perto, relacionadas com a tomada de decisão do próprio paciente (Maier et al, 2020). Assim, como o elemento 36 apresentava cárie e doença periodontal com mobilidade grau 3 foi necessário à sua extração. 
Vieira et al, (2019) afirma que o processo contínuo de movimentação de um dente do seu local de desenvolvimento para sua posição funcional é denominado de erupção, uma vez chegada a época normal em que deveriam irromper, ficam encerrados no interior do osso, podem ter ou não o folículo pericoronário. A retenção dentária é, geralmente, de ordem mecânica, impacção, podendo ser também um posicionamento atípico do germe dentário, anquiloses, formações císticas e neoplásicas, trauma dentoalveolar, dilaceração radicular, doenças sistêmicas e síndromes. É possível observar a princípio um caso clínico em que ao exame de imagem há a presença de capuz pericoronário, o que poderia em algum momento propiciar o desenvolvimento de lesão cística ou tumoral de origem odontogênica. Além disso, Costa (2018) afirma que os tumores e cistos odontogênicos originados dos tecidos de formação do órgão dental apresentam ocorrência relativamente comum nos maxilares.

Pode-se perceber o envolvimento da coroa do dente 35, impactado, na região de defeito periodontal do dente 36, condenado devido à extensa lesão cariosa envolvendo até a raiz e significativa mobilidade às custas de perda óssea. Além disso, é notável que a programação de tracionamento ortodôntico após a exodontia do dente 36, comprometido, requer tecido ósseo suficiente para ancoragem e o investimento nessa abordagem possui grandes chances de fracasso, havendo assim um custo benefício duvidoso e prognóstico desfavorável. As causas mais comuns para profissionais não indicarem o tracionamento ortodôntico são: proximidade das raízes adjacentes, podendo causar reabsorção radicular lateral nos incisivos laterais e nos prémolares, reabsorção cervical externa nos caninos tracionados, anquilose alvéolo-dentária do dente envolvido, metamorfose cálcica da polpa e necrose pulpar asséptica (Frank, 2000).

A exodontia do dente 36 foi realizada sem dificuldades pela via alveolar, o que expos parcialmente a coroa do elemento 35. Porém, mesmo assim foi necessário a realização de ostectomia, a fim de permitir a criação de sítios de apoio para o uso dos extratores, além da odontosecção, que se tornou necessária, uma vez que o dente 35 não apresentava um eixo de saída favorável. Ferreira-Filho et al, (2021) afirma que, para um bom pós-operatório e livre de complicações pós-cirurgia, é essencial equilibrar a quantidade de osso removida durante exodontia, por isso é importante respeitar a posição e o sentido longo eixo do dente durante realização da odontosecção a fim de uma abordagem efetiva. Então, o elemento dentário deverá ser extraído com auxílio de um extrator reto, realizando uma leve pressão no espaço desgastado, assim também como a osteotomia.

Um fator de importante destaque é a discreta parestesia temporária do nervo mentual esquerdo apresentada pela paciente. Aquino, Rocha, Lima, Araújo e Ramos Oliveira (2020) afirma que na prática da cirurgia oral os acidentes e complicações mais comuns que podem acontecer no transoperatório e pós-operatório destaca-se as lesões nervosas que resultam em parestesia. Esta complicação pode ser definida como uma lesão nervosa caracterizada pela perda de sensibilidade, transitória ou permanente do nervo afetado, de acordo com De Lima et al, (2019), a prevalência dessa complicação pós exodontias de dentes inferiores, no nervo alveolar inferior é de 18,6\% e no nervo lingual é de 7,0\%. A parestesia pode acontecer principalmente devido à proximidade da raiz dentária com o nervo e à posição e nível de angulação da área operada. Como o paciente sentiu apenas uma discreta parestesia durante 30 dias com melhora gradativa, sabe-se que ela é apenas transitória.

Assim, mesmo sabendo que o tracionamento ortodôntico, é uma terapêutica mais conservadora e pode estar associada à remoção cirúrgica de uma pequena parte da mucosa, exposição do elemento dentário e o seu tracionamento, ou até mesmo a sua erupção espontânea a decisão de extrair ou manter o dente e utilizar manobras ortocirúrgicas requer avaliação anatômica, como angulação do dente, espaço disponível no arco e proximidade radicular, já que atrelado a isso os tratamentos podem se tornar mais longos e procedimentos menos conservadores passam a serem considerados para atenuar a morbidade (Rocha, de Jesus Silva, \& Souza, 2020). Antes de qualquer tratamento, deve-se levar em consideração: idade da paciente, desvitalização da unidade tracionada, reabsorções radiculares, a anquilose alvéolo-dentária, a proximidade das coroas, bem como das raízes adjacentes e o tempo / custo do tratamento. (Frank, 2000) levando em consideração ao tempo e custo que a paciente teria que arcar, foi decidido junto a ela, a extração de ambos. Dessa forma, o procedimento cirúrgico foi finalizado com reposicionamento do retalho e sutura, orientações e prescrição medicamentosa. Dessa forma, pode-se afirmar que a parestesia transitória observada 
no pós-operatório não foi causada por dano direto significativo ao nervo.

\section{Conclusão}

O procedimento cirúrgico para exodontia do elemento impactado, neste caso, foi de significativa relevância, uma vez ponderado seus riscos e benefícios em relação as demais opções de tratamento. Sendo assim, a estratégia de abordagem utilizada apresentou sucesso na condução do caso clínico. Tal abordagem permite a futura discussão de casos clínicos semelhantes, onde opções alternativas de tratamento sejam adotadas.

\section{Referências}

Abu-Hussein, M., Watted, N., Emodi, O., \& Awadi, O. (2015). Management of lower second premolar impaction. Journal of Advanced Dental Research, 1(1), 71-79. http://nebula.wsimg.com/668ed4aca3a7b378d200f4ef8ff2e54f?AccessKeyId=E54D0FD2D82F47860512\&amp;disposition=0\&amp;alloworigin=1

Al-Abdallah, M., AlHadidi, A., Hammad, M., \& Dar-Odeh, N. (2018). What factors affect the severity of permanent tooth impaction?. BMC oral health, 18(1), 184. https://doi.org/10.1186/s12903-018-0649-5

Allareddy, V., Caplin, J., Markiewicz, M. R., \& Meara, D. J. (2020). Orthodontic and surgical considerations for treating impacted teeth. Oral and Maxillofacial Surgery Clinics of North America, 32(1), 15-26. https://doi.org/10.1016/j.coms.2019.08.005

Alling, C. C., \& Catone, G. A. (1993). Management of impacted teeth. Journal of Oral and Maxillofacial Surgery, 51(1), 3-6. https://doi.org/10.1016/02782391(93)90004-w

Aquino, T. S. d., Rocha, A. D. O., Lima, T. O., Araujo, T. M. R., \& Ramos Oliveira, T. M. (2020). Laserterapia de baixa potência no tratamento de parestesia oral - uma revisão sistematizada. Revista Eletrônica Acervo Odontológico, 1, Artigo e3753. https://doi.org/10.25248/reaodonto.e3753.2020

Brown, K., Cheah, T., \& Ha, J. F. (2016). Spontaneous cutaneous extrusion of a parotid gland sialolith. BMJ case reports, 2016 , bcr2016214887. https://doi.org/10.1136/bcr-2016-214887

Burch, J., Ngan, P., \& Hackman, A. (1994). Diagnosis and treatment planning for unerupted premolars. Pediatric dentistry, 16(2), 89-95.

Clark C. A. (1910). A Method of ascertaining the Relative Position of Unerupted Teeth by means of Film Radiographs. Proceedings of the Royal Society of Medicine, 3(Odontol Sect), 87-90.

Collett A. R. (2000). Conservative management of lower second premolar impaction. Australian dental journal, 45(4), 279-281. https://doi.org/10.1111/j.18347819.2000.tb00264.x

Costa, D. C. B. (2018). Estudo da ocorrência de recidivas após enucleação, seguida de ostectomia periférica e solução de carnoy no tratamento das lesões odontogênicas benignas agressivas [Dissertação de mestrado, Universidade Federal do Rio Grande do Norte]. https://repositorio.ufrn.br/bitstream/123456789/27024/1/Estudoocorrênciarecidivas_Costa_2018.pdf

de Lima, N. M., Sampaio, L. T. R., Alves Filho, M. E. A., Barreto, J. O., Freire, J. C. P., Rocha, J. F., \& Ribeiro, E. D. (2018). Complicações associadas à exodontias de terceiros molares: um estudo de prevalência. Archives of health investigation, 7.

Duarte, F., Figueiredo, R., Ramos, C., Esteves, H., Salazar, F., Martins, M., \& Figueira, F. (2005). Inclusão de dentes pré-molares. Separata Científica do HSOSA, 1(7), 4-8. https://www.clitrofa.com/PublicacoesCientificas/CirurgiaOral/Inclusao_de_Dentes_Pre-Molares.pdf

Ferraz, T. M., Carneiro, L. S., Stecke, J., Rayes, N., \& Oliveira, G. B. D. (2019). Achados na radiografia panorâmica indicam tomografia computadorizada no pré-operatório de terceiro molar inferior: Relato de caso. Revista Odontológica do Brasil Central, 28(84). https://doi.org/10.36065/robrac.v28i84.1299

Ferreira Filho, M. J. S., Neto, I. C. B., da Penha Melo, L., do Vale, W. H. S., Corrêa, A. K., Aguiar, F. M., \& Milério, L. R. (2021). A importância da técnica de odontosecção em exodontia de terceiros molares: revisão de literatura. Brazilian Journal of Development, 7(2), 13100-13112.

Filho, J. D. S. F., França, S. R., Araújo, L. K., Pereira, J. J. d. N., Belchior, I. F. C., \& Sampieri, M. B. D. S. (2018). Intervenção cirúrgica de um canino incluso em sínfise mandibular: Relato de caso. Revista Da Faculdade De Odontologia - UPF, 23(3), 329-332. https://doi.org/10.5335/rfo.v23i3.8613

Frank C. A. (2000). Treatment options for impacted teeth. Journal of the American Dental Association (1939), 131(5), 623-632. https://doi.org/10.14219/jada.archive.2000.0236

Infante-Cossio, P., Hernandez-Guisado, J. M., \& Gutierrez-Perez, J. L. (2000). Removal of a premolar with extreme distal migration by sagittal osteotomy of the mandibular ramus: report of case. Journal of oral and maxillofacial surgery : official journal of the American Association of Oral and Maxillofacial Surgeons, 58(5), 575-577. https://doi.org/10.1016/s0278-2391(00)90026-0

Kaczor-Urbanowicz, K., Zadurska, M., \& Czochrowska, E. (2016). Impacted Teeth: An Interdisciplinary Perspective. Advances in clinical and experimental medicine : official organ Wroclaw Medical University, 25(3), 575-585. https://doi.org/10.17219/acem/37451

Maier, J., Sfreddo, C. S., Reiniger, A. P. P., Zanini Kantorski, K., Wikesjö, U. M., \& Moreira, C. H. C. (2020). Residual periodontal ligament in extracted teeth - is it associated with indication for extraction? International Dental Journal. https://doi.org/10.1111/idj.12621 
Research, Society and Development, v. 10, n. 14, e257101421939, 2021

(CC BY 4.0) | ISSN 2525-3409 | DOI: http://dx.doi.org/10.33448/rsd-v10i14.21939

Mohammed, M., Mahomed, F., \& Ngwenya, S. (2019). A survey of pathology specimens associated with impacted teeth over a 21 -year period. Medicina oral, patologia oral y cirugia bucal, 24(5), e571-e576. https://doi.org/10.4317/medoral.22873

Primo, B. T., Andrade, M. G. S., de Oliveira, H. W., \& de Oliveira, M. G. (2011). Dentes retidos: novas perspectivas de localização. Revista da Faculdade de Odontologia-UPF, 16(1).

Rocha, L. M. D. S. R., de Jesus Silva, F., \& Souza, G. A. (2020). Critérios para decisão do tratamento de caninos inclusos: Exodontia versus Tracionamento. Brazilian Journal of Health Review, 3(6), 15872-15878.

Sandler, P. J., \& Springate, S. D. (1991). Unerupted premolars--an alternative approach. British journal of orthodontics, 18(4), 315-321. https://doi.org/10.1179/bjo.18.4.315

Valente, N. D. A., Soares, B. M., Santos, E. J. D. C., \& Silva, M. B. F. (2016). A importância da TCFC no diagnóstico e localização de dentes supranumerários. Revistas, 73(1), 55. https://doi.org/10.18363/rbo.v73n1.p.55

Vieira, V. V., de Assis, B. L. P., Carrizo, D. P. F., Bueno, J. M., Gomes, C. C., \& Mundim-Picoli, M. B. V. (2019). Diagnóstico de dente retido associado a lesão cística através de tomografia computadorizada por feixe cônico: Relato de caso. Anais da Jornada Odontológica de Anápolis-JOA. 